\title{
Forensic DNA Analysis
}

\author{
Christian Gehrig* and Anne Teyssier
}

\begin{abstract}
Currently the method of choice in the field of forensic identity is the use of commercially available kits allowing the simultaneous analysis of several STRs in a single multiplex reaction. Several other technologies, like the sequencing of mitochondrial DNA and typing of markers on the $Y$ chromosome are providing forensic DNA laboratories with a new arsenal of weapons that may be used in the field of human identification. Switzerland has implemented a forensic DNA database with already remarkable results.
\end{abstract}

Keywords: DNA databases $\cdot \mathrm{mtDNA} \cdot \mathrm{PCR} \cdot \mathrm{STR} \cdot \mathrm{Y}-\mathrm{STR}$

\section{Introduction}

The advent of modern DNA technology has resulted in the increased ability to perform human identity testing. DNA typing is useful in a number of situations including the determination of perpetrators of violent crime such as murder and rape, resolving questions of paternity, historical investigations, identifying remains of missing persons or victims of mass disasters, and forensic DNA databases. The aim of this article is to describe the use of different DNA typing techniques in the field of human identification.

\section{History of Forensic DNA Analysis}

A little more than a decade ago, all the forensic genetic casework was performed using classical serological genetic markers. Blood groups, HLA and polymorphic protein and enzymes were used for solving forensic genetic casework using immunological and electrophoretic methodologies. These genetic markers were nevertheless limited when it was necessary to analyze minimal or degraded material, which is commonly involved in forensic cases. It was, in addition, difficult to analyze biological material other than blood, and therefore the information obtained from hair, saliva and even semen in rape cases was rather limited.

The discovery of polymorphisms in repetitive DNA by Jeffreys et al. [1] in 1985 has had a tremendous impact on forensic genetics. In the last ten years, highly informative and robust DNA typing systems have been developed which are quite powerful for the individualization of biological material of human origin.

\section{Short Tandem Repeat (STRs) Markers}

DNA regions with repeat units that are 2-6 bp in length are called microsatellites or short tandem repeats (STRs) (Fig. 1). STRs have become popular markers because they can be easily amplified by the polymerase chain reaction (PCR). The number of repeats in STR markers can be highly variable among individuals, which makes them effective for human identification purposes.

\section{Typing by PCR Multiplex}

For DNA typing to be effective in the forensic community, a common set of standardized markers must be used. In 2000 the Swiss Society of Legal Medicine adopted the SGM Plus system, which is widely used in neighboring countries, as its choice for DNA typing of crime scene material. This commercially available kit permits the robust multiplex

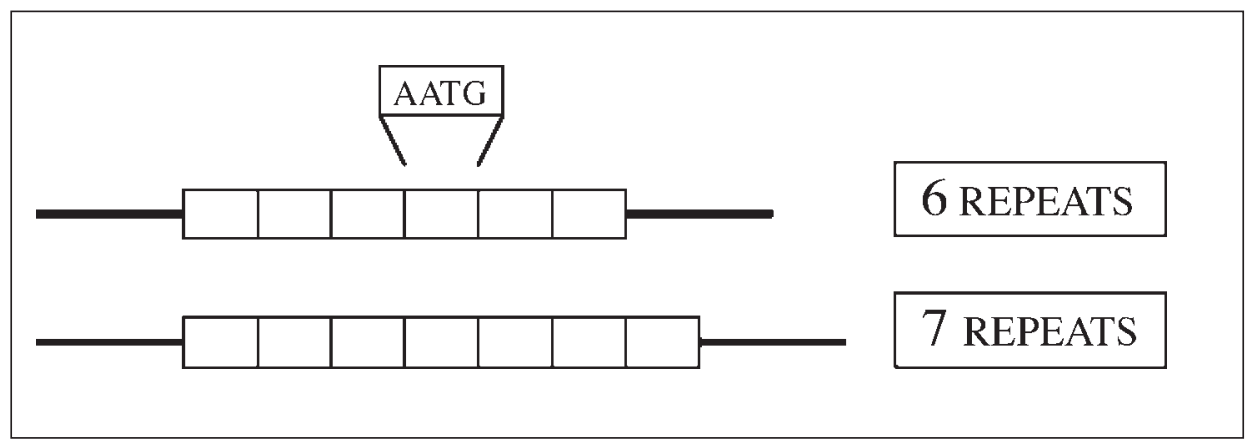

Fig.1. Schematic representation of a STR marker. 
amplification of 10 STR markers and a gender identification marker in a single reaction with $1 \mathrm{ng}$ (or less) of DNA sample. Over the years a number of methods has been used for detecting DNA molecules following electrophoretic separation. Almost all commercially available STR typing kits involve the use of fluorescent-labeled PCR primers. Amplified STR alleles are visualized as bands on a gel or represented by peaks on an electropherogram (Fig 2.).

The genetic profile can be transformed into an alphanumerical code starting with the letters XY (in case of male DNA) or XX (in case of female DNA), followed by a combination of twenty numbers (Fig. 3). This combination of numbers is, with the exception of identical twins, practically unique for each human being. The frequency of a typically SGM Plus profile is extremely rare in the order of one in several billion individuals.

\section{Casework}

In the following case, two suspects (A and $\mathrm{B}$ ) and semen on a vaginal swab from a rape case were typed with the SGM Plus kit (Fig. 4). Suspect A shows a different profile than that of the semen and can therefore be excluded as the donor of the semen. On the other hand suspect B cannot be excluded. How is the result to be interpreted for suspect B [2]? There are only two possibilities: suspect B provided the semen or someone else provided the material. The calculations are simplest when 'someone else' refers to a person unrelated to the suspect, but specified

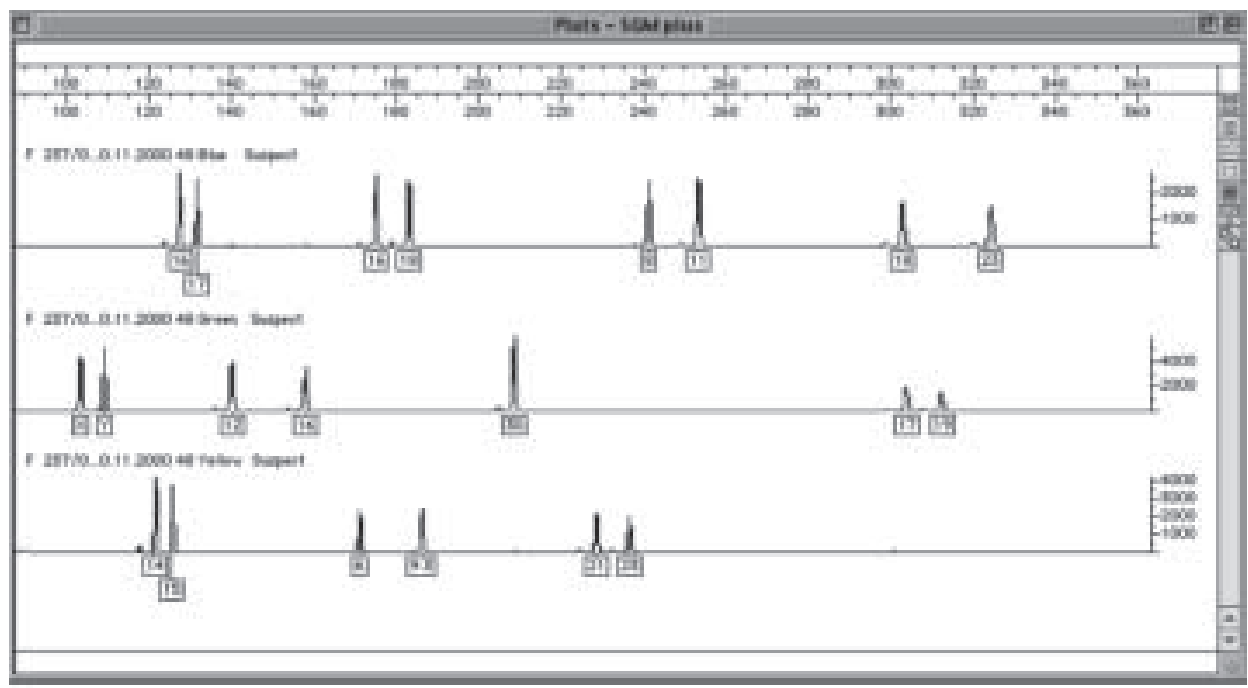

Fig. 2. SGM Plus profile

$\mathrm{XY} / 16-17 / 16-18 / 8-11 / 18-23 / 12-16 / 30-30 / 17-19 / 14-15 / 6-9.3 / 21-23$

Fig. 3. Alphanumerical code of a SGM plus profile

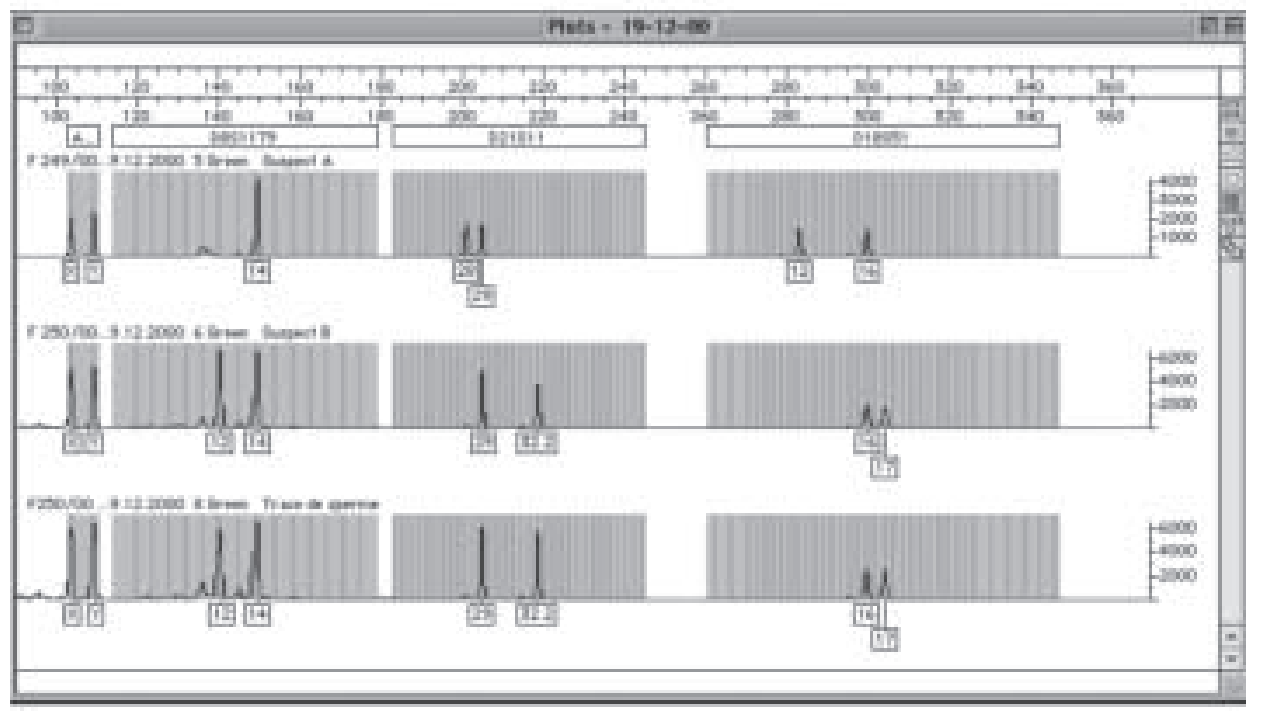

degrees of relationship can also be accommodated. For each of these two scenarios, two probabilities can be calculated and compared by the means of a likelihood ratio (LR). In this case:

$$
\begin{array}{ll}
\mathrm{LR}=1 / \mathrm{p} & \mathrm{p}: \text { frequency of the profile } \\
\text { in the population }
\end{array}
$$

With the SGM Plus system, the LR is extremely high, typically greater than 1 billion, and the evidence is presented in the form: 'The evidence is 1 billion times more probable if suspect B left the semen stain than if some unknown unrelated person left the semen stain'. Some experts prefer to report only the random match probability which is the ' $p$ ' defined above. In mixture cases, the evidence must be evaluated through a likelihood ratio [3].

\section{Paternity Testing}

The often obvious resemblance between a child and his parents is due to the fact that a child receives half of his genetic information from his biological mother and the other half from his biological father. Generally between 10 and 15 STR markers are analyzed in a paternity case. During the testing process, the genetic characteristics of a child are first compared to those of its mother. Those characteristics that cannot be found in the mother must have been inherited from the biological father. If the tested man does not contain the genetic characteristics necessary to be the biological father of the child, he is excluded. If the DNA of the tested man does contain those genetic characteristics (Fig. 5), then this man cannot be excluded and the probability that this man is the true biological father can be calculated. The probability of paternity is generally greater than $99.99 \%$. The paternity is 'practically proven'.

\section{Mitochondrial DNA (mtDNA) Analysis}

The vast majority of the human genome is located within the nucleus of each cell. However, mitochondria, which are located in the cytoplasm and provide the energy of the cell, contain a small circular genome consisting of 16569 bp. A non-coding 'control region' exhibits a

Fig. 4. The gender of the three samples analyzed (suspect $A$, suspect $B$ and the semen) and three of the ten SGM Plus loci analyzed. 


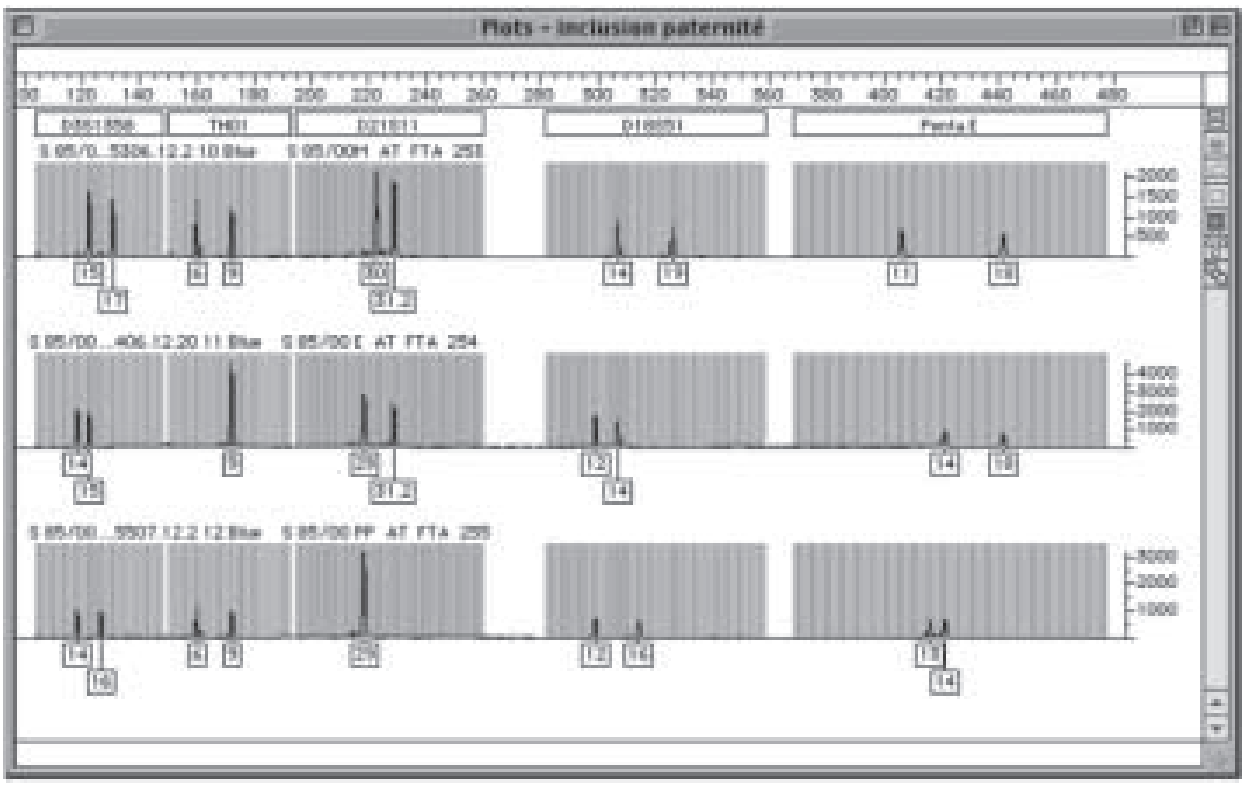

fair degree of variation between individuals and is therefore useful for human identity testing purposes. MtDNA is valuable to the forensic community because it can be efficiently amplified from limited or severely degraded biological material, typically bones and hair [4].

Human mtDNA is inherited strictly from one's mother. The consequence in forensics is that an individual's mother, siblings, and all other maternally related family members should have, barring a mutation, identical mtDNA sequences. Identity may therefore be determined by comparing mtDNA sequences of an unknown sample to sequences from any maternal reference, even if the unknown and reference individuals are separated by many generations. This fact can be helpful in solving missing persons or mass disaster investigations but can reduce the significance of a match in forensic cases.

\section{Y-Chromosome STRs}

Y-chromosomal STR polymorphisms are becoming of increasing interest in the forensic field [5], because of their possible applications in stain analyses and paternity testing in male lineage cases. Since the majority of sexual offenses have a male perpetrator and a female victim, using Y-chromosome markers can improve the chances of being able to detect small amount of male perpetrator DNA in a high background of heterologous female DNA.

Statistical interpretation in cases of a match is, the same as for mtDNA, more complicated and appropriate corrections taking into account population substructure and sampling errors need to be per- formed. Population compilations are therefore very important and some efforts have been done regarding this. Y-haplotype data of several thousands of male individuals have been typed in a worldwide effort, the data is available at www.ystr.org.

\section{Swiss National DNA Database}

On July 1, 2000, the Swiss Federal Council (Swiss Government) set the regulation for the DNA Profile Information System that has been operational since August 1, 2000. The regulation contains a specified catalogue of crimes (from burglary to murder) that regulates the admittance of DNA profiles from suspects. Buccal swabs are taken from suspects by the police. These samples and crime scene material are then analyzed by the local DNA laboratory at the Institutes of Legal Medicine either in Basel, Bern, Geneva, Lausanne, St. Gallen or Zürich. The police anonymizes all samples with a unique identification number (PCN). At the same time they transfer the name of the suspect, his/her fingerprints as well as other relevant information to the AFIS services of the federal police at Bern. The forensic genetic laboratories transmit the DNA profiles to the DNA Coordination Unit at the Institute of Legal Medicine at Zürich that imports the profiles into the DNA database. The database system chosen by Switzerland is the COmbined DNA Index System or CODIS, developed by the Federal Bureau of Investigation (FBI). Daily automated searches are performed and so called hits - identical DNA profiles between stains or stains and persons - are reported to the AFIS services by only providing the relevant
Fig. 5: The genetic profiles (top to bottom: mother, child, and alleged father) for five STR markers. The alleged father cannot be excluded for any of the five markers.

PCN numbers. The AFIS services link the PCN numbers with the corresponding names and crimes and inform the investigating police unit about the hit. By this procedure a strict separation of DNA data and other personal data is achieved.

By the end of February 2002, approximately 15168 profiles from suspects and 2227 from crime scene stains were gathered and stored in the Swiss DNA database. These databases can be used to identify suspects in crime cases that would otherwise never have been solved. So far 748 matches linking individuals to crime scenes were observed. A second important role of this database is that it can serve to make associations between groups of unsolved cases. So far 402 matches linking crime scene to crime scene have been observed and therefore providing the police with an investigative lead that would otherwise not have developed.

The largest group of crimes linked by matches were burglaries followed by offences like endangering life and crimes against sexual integrity. A federal DNA law is in preparation and should harmonize the legal situation for the whole country and replace the current regulation that only legalizes the procedure until the end of 2004.

In conclusion, the incorporation of modern molecular biological techniques (multiplex STRs, mtDNA and Y-chromosome markers) in the laboratory has resulted in the identification, with a very high degree of certainty, of biological fathers, of criminals and also the exoneration of the innocent.

Received: January 22, 2002

[1] A.J. Jeffreys, V. Wilson, S.L. Thein, 'Hypervariable 'minisatellite' regions in human DNA', Nature 1985, 316, 76.

[2] I.W. Evett, B.S. Weir, 'Interpreting DNA evidence', Sinauer and Associates 1998.

[3] T. Clayton, J. Whitaker, R. Sparkes, P. Gill, 'Analysis and interpretation of mixed forensic stains using DNA STR profiling', $F_{O}$ rensic Sci. Int. 1998, 91, 55-70.

[4] M. Holland, T. Parson, 'Mitochondrial DNA sequence analysis - validation and use for forensic casework', Forensic Sci. Rev. 1999, 11, 21-50.

[5] C. Gehrig, M. Hochmeister, B. Budowle, 'Swiss allele frequencies and haplotypes of 7 Y-specific STRs', J. Forensic Sci. 2000 , 45(2), 436-439. 